\title{
pro.posições
}

ISSN 1980-6248

10.1590/1980-6248-2015-0010

ARTIGOS

\section{Ampliaciones y desigualdades en el acceso y egreso de estudiantes a la Educación Superior en la Argentina}

Expansões e desigualdades no acesso e egresso dos alunos para o ensino superior na Argentina

\section{Expansions and inequalities in student's access and graduation to higher education in Argentina}

Claudio Suasnábar*

Laura Ines Rovelli**

*Universidad Nacional de La Plata, Instituto de Humanidades y Ciencias Sociales, Buenos Aires, Argentina.csuasnabar@gmail.com

**Universidad Nacional de La Plata, Facultad de Humanidades y Ciencias de la Educación; CONICET

- IdHCS. Buenos Aires, Argentina. laurarovelli@gmail.com

\begin{abstract}
Resumen
El presente artículo analiza los cambios en la composición social de la matrícula de educación superior operados en el período 2004-2012 en la Argentina, con especial énfasis en la caracterización socioeconómica de la población estudiantil. Por una parte explora las relaciones entre educación universitaria y no universitaria (terciario) y por otra, entre las dinámicas de expansión del nivel medio y superior. La estrategia metodológica de la investigación es de corte cualitativa complementada por el uso de fuentes estadísticas. La hipótesis del trabajo plantea que los sistemas "amplios" de acceso a la educación superior, como los de la Argentina, configuran dinámicas de expansión y selectividad para el acceso a la misma que se relacionan fuertemente con las lógicas de ampliación de la matrícula en el nivel secundario y con la conformación de circuitos de educación diferenciados según los ingresos.

Palabras clave: ampliación, desigualdades, acceso, egreso, educación superior .
\end{abstract}




\section{Resumo}

Este artigo analisa as mudanças na composição social das matriculas no ensino superior operados no período 2004-2012, na Argentina, com especial ênfase na caracterização socioeconômica da população estudantil. Por uma parte, explora a relação entre educaşão universitária e não universitária (ensino terciario), e por outra parte, a dinâmica de expansão entre Ensino Médio e Ensino Superior global (universitária e não universitária). A estratégia metodológica da pesquisa é de tipo qualitativo, complementada com fontes estatísticas. A bipótese de trabalbo propõe que os sistemas de acesso "mais abertos" de Ensino Superior, como na Argentina, têm uma dinâmica de expansão e seletividade no acesso que se relacionam fortemente com a lógica da expansão do Ensino Médio e a configuração de circuitos de educação diferenciada de acordo aos niveis de renda.

Palavras-chave: extensão, desigualdades, acesso, graduação, ensino superior

\section{Abstract}

This article analyzes the changes in the social composition of enrollment in Higher Education operated in the period 2004-2012 in Argentina, with special emphasis on the socio-economic characterization of the student population. On the other hand, explores the relationship between university and tertiary education on the one band and between the dynamic expansion of secondary and higher The methodological strategy is qualitative research complemented by cutting the use of statistical sources. The working hypothesis states that the "large" systems of access to Higher Education, such as Argentina, configure dynamic expansion and selectivity for access to Higher Education that relate strongly with the logic of expanding enrollment in the secondary level and the circuit configuration of differentiated education based on income.

Keywords: expansion, inequalities, access, graduation, higher education 


\section{pro.posições}

\section{Presentación}

En las últimas cuatro décadas, la expansión de la educación superior en América Latina constituye una poderosa tendencia que con diferentes ritmos abarca al conjunto de los países de la región. Siguiendo la tradicional clasificación de Martín Trow (2005) podemos decir que la educación superior en la región ha dejado atrás la etapa de sistemas de élite (menos del $15 \%$ de tasa de escolarización) para ubicarse en el tránsito de sistemas de masas (entre 15 y 50 \%) hacia la universalización de este nivel (más del 50\%).

Los últimos quince años marcan -en particular- el inicio de un segundo ciclo de expansión de la educación superior que, a diferencia del primero operado en las décadas del 1960 y 1970 donde la centralidad de la agenda de política estuvo dada por la idea de modernización, realiza especial énfasis en la cuestión de la ampliación e inclusión. Lo anterior se manifiesta en el reconocimiento del carácter de bien público de la educación superior (CRES, 2008) y en las diferentes estrategias de políticas impulsadas en cada país. Con todo, las dinámicas de expansión de la educación conviven con rasgos estructurales que delinean su carácter contradictorio y desigual, por lo que asegurar una plena democratización de la educación superior todavía constituye un desafío para las políticas estatales y las propias instituciones universitarias.

Así, la centralidad de la inclusión educativa en la agenda de política de la educación superior expresa la toma de consciencia de los gobiernos respecto de que la ampliación del acceso no necesariamente garantiza la democratización de este nivel. Siguiendo a Chiroleu (2012), la problematización en términos formales de la igualdad o ampliación del acceso persigue habitualmente la expansión de la matrícula aunque no implica necesariamente la representación equitativa de los diversos grupos sociales en el conjunto de la población de la educación superior, siendo esta última una cuestión propia de la búsqueda de la democratización del nivel. En cierta medida, la concepción de políticas en torno a la igualdad del acceso lleva a la distribución de bienes educativos en pos del desplazamiento del umbral educativo de las naciones, lo cual supone concebir a las instituciones como espacios neutrales en términos de las posiciones y disputas sociales (Dubet, 2012). En contraste, el concepto de inclusión parte de la aceptación de las desigualdades existentes y procura revalorizar la diversidad social y reducir las discriminaciones existentes a través de la igualdad de oportunidades (Chiroleu, 2012; Dubet, 2012). 


\section{pro.posições}

Esta preocupación resulta perceptible en las políticas implementadas en los años recientes en Brasil donde la creación de nuevas universidades se combina con políticas de afirmación positiva a través de cuotas en el ingreso y becas en el sector privado para ampliar las plazas; en Uruguay a través de una estrategia de descentralización de la Universidad de la República hacia la generación de polos de desarrollo universitario o en Venezuela donde la ampliación del acceso supuso la creación de nuevos tipos de instituciones a través de modelos innovadores que sin modificar las universidades tradicionales, amplían las oportunidades de acceso al nivel superior.

Paralelamente en la Argentina, con especial énfasis entre 2008 y 2015, un conjunto de programas de becas lanzados o reformulados desde el Ministerio de Educación de la Naciónnos referimos al Programa Nacional de Becas Universitarias (PNBU), al Programa de Becas del Bicentenario (PNBB) y al Programa de Respaldo a Estudiantes de Argentina (PROG.R.ES.AR)- se orientan hacia las demandas de democratización de segunda generación. Es decir, basados en el principio de igualdad de oportunidades, apuntan en principio a generar una igualdad de resultados a partir de la desigualdad de tratamientos ${ }^{1}$.

En este marco, el presente escrito propone dos líneas de indagación: la primera focaliza el interés en el análisis de los cambios en la composición social de la matrícula de educación superior operados en el período 2004-2012 en la Argentina, con especial énfasis en la caracterización socioeconómica de la población estudiantil. La segunda línea de indagación explora, por un lado, las relaciones entre educación universitaria y educación no universitaria (terciaria) y por otro, las dinámicas de expansión del nivel medio y la educación superior tomadas globalmente. La estrategia metodológica de la investigación es de corte cualitativa complementada por el uso de fuentes estadísticas. Éstas surgen de la Encuesta Permanente de Hogares (EPH) del Instituto Nacional de Estadística y Censos de la Argentina (INDEC), la cual por sus características posibilita relevar, sistematizar y analizar las distintas dimensiones y tendencias de cambio de las dinámicas de expansión y las características de la población

1El PNBU se dirige a garantizar la igualdad de oportunidades para ingresantes y estudiantes avanzados; el PNBB privilegia estrategias de permanencia y egreso para estudiantes e incorpora la jerarquización de ciertas carreras consideradas prioritarias para el desarrollo nacional; mientras que el PROG.R.ES.AR constituye un apoyo económico para jóvenes de entre 18 y 24 años que quieran iniciar, reiniciar o finalizar estudios en algún nivel del sistema educativo, el cual es complementado por dispositivos de inclusión como tutorías, guarderías para aquellos que tengan hijos e inserción laboral de estudiantes. 


\section{pro.posições}

estudiantil y de los Anuarios de Estadísticas Educativas y Universitarios del Ministerio de Educación de la Nación2.

La hipótesis del trabajo plantea que los sistemas "amplios" de acceso a la educación superior, como los de la Argentina, configuran dinámicas de expansión y selectividad para el acceso a la educación superior que se relacionan fuertemente con la lógica de ampliación de la matrícula en el nivel secundario preexistente y con la configuración de circuitos de educación diferenciados según los ingresos - aunque no solamente- de los jóvenes3. Por sistemas "amplios" nos referimos a la modalidad de acceso más extendida en las universidades públicas argentinas basada en el ingreso directo, sin cupo o examen de ingreso eliminatorio, aunque no desconocemos la proliferación en las últimas décadas de dispositivos más selectivos en algunas instituciones y al interior de ellas, en ciertas unidades académicas (Duarte, 2005).

En adelante, en el primer apartado el trabajo presenta un breve estado del arte sobre la temática abordada; en el segundo se analizan las lógicas de expansión de matrícula de la educación superior y el crecimiento desigual entre el circuito universitario y el no universitario (terciario). La tercera sección explora la evolución del acceso y egreso de estudiantes de la Educación Superior según quintil de ingreso per cápita de los hogares argentinos con el propósito de evaluar en qué medida el sistema se ha ido abriendo a los grupos de menores ingresos de la sociedad. El cuarto apartado desarrolla la relación entre la matrícula de la escuela media y el acceso a la educación superior. Por último se introducen algunas consideraciones finales en torno a la investigación.

\section{Breves notas sobre educación y desigualdad en el nivel superior}

La relación entre educación y equidad social es una problemática clásica de la sociología de la educación que también acaparó buena parte de los debates políticoacadémicos internacionales de las décadas de 1960 y 1970. Las corrientes desarrollistas y la teoría del capital humano plantearon inicialmente una relación directa entre mayor educación y disminución de las desigualdades. Al respecto, Raymond Boudon (1974) reveló que, si bien la

2La información presentada en este escrito surge a partir de la selección de una muestra representativa por parte de la Encuesta Permanente de Hogares, en determinadas zonas de aglomerados encuestados.

3 Entendemos que otras dimensiones sociales y culturales juegan un papel igual o mayor en importancia que las económicas pero escapan a las posibilidades de este estudio. 


\section{pro.posıções}

igualdad de oportunidades educativas ofrecidas a los sectores populares supone un mayor acceso al conocimiento, el mismo no se traduce en la disminución de la brecha social respecto de los sectores altos de la población, por cuanto el carácter piramidal de la estructura social restringe las posibilidades de ascenso social. A su vez, Randall Collins (1989) mostró el peso diferencial de las credenciales educativas para el acceso a los puestos de trabajo y cómo el mercado laboral resulta un mayor condicionante que la posesión de credenciales. Y finalmente los estudios de Pierre Bourdieu (1970) y Pierre Bourdieu y Jean-Claude Passeron (2003) avanzaron en la comprensión macro y micro social de los procesos de reproducción a partir del peso del capital cultural de origen en la determinación de éxito y fracaso de los distintos sectores. En un contexto marcado por la expansión del Estado de Bienestar en los países centrales, estos debates plantearon una crítica a las expectativas y promesas de la expansión estatal.

En el contexto de la crisis mundial de los últimos años se renuevan estos debates poniendo en primer plano la cuestión de la igualdad de oportunidades y la igualdad de posiciones (Dubet, 2010). Así, si la primera supone la apuesta por generar las mismas posibilidades de la población para acceder a la educación y la competencia meritocrática, lo cual legitima ciertas "desigualdades justas", la segunda postula la necesidad de disminuir la brecha entre las posiciones sociales aunque esto conlleve una menor o inexistente movilidad social. Por su parte, la búsqueda de igualdad de resultados se asienta sobre el principio de la diferencia y busca poner un límite a las desigualdades propias de la competencia meritocrática, al privilegiar políticas que limiten las brechas entre el nivel de educación, el tipo de establecimientos y los circuitos educativos al que acceden los más débiles y los más favorecidos socialmente (Dubet, 2012). Se trata en definitiva de tres modelos de justicia educativa internos a los sistemas educativos que buscan la utilidad razonable en la distribución de diplomas en las sociedades democráticas modernas.

Desde una dimensión institucional sobre los problemas abandono/deserción universitaria en los primeros años existe una amplia producción principalmente en Estados Unidos. En este país la masificación de la educación superior a través de los colleges y community colleges presenta altas tasas de abandono, por lo cual desde la década de 1980 la temática pasó a ocupar un lugar central en la agenda de políticas de las instituciones y se traduce en la generalización de dispositivos y programas orientados a los primeros años (Barefoot, 2000, 2005; Tinto, 2004). 


\section{pro.posıções}

Alrededor de estas cuestiones buena parte de la investigación actual en la Argentina y a nivel regional ponen especial atención al estudio de las políticas de ampliación del acceso, y en particular, aquellas orientadas a garantizar la permanencia y egreso de sectores anteriormente excluidos de la educación superior (Aponte-Hernández, 2008; Chiroleu, 2009a, 2009b; Kessler, 2014; Siteal, 2005).

Precisamente, la educación constituye una de las áreas que más claramente revelan la tensión entre la expansión de la educación y persistencia de desigualdades o, dicho de otra manera, que el aumento de la matrícula global supone un acceso diferencial (permanencia y graduación) de los sectores sociales a los distintos niveles del sistema y también un acceso diferencial al conocimiento.

\section{Expansión de la matrícula y diversificación de las instituciones de la Educación Superior: crecimiento desigual y diferenciación institucional}

La configuración histórica de la educación superior en la Argentina está marcada por el predominio de la universidad pública hasta la mitad del siglo XX. Así, el origen de la universidad se sitúa en 1613 con la fundación de la Universidad de Córdoba en el período colonial, posteriormente en el período pos independentista se crea la Universidad de Buenos Aires en 1812 y en las primeras décadas del siglo XX se crean las Universidades de La Plata (1905) y de Tucumán (1914). En el contexto de la crisis del orden oligárquico y de democratización política, la Reforma Universitaria de 1918 establece los principios de autonomía y co-gobierno que progresivamente se constituye como identidad de la comunidad universitaria.

En las décadas posteriores y durante el gobierno peronista se fundan nuevas universidades públicas y recién hacia finales de la década de 1950 se crearán las primeras universidades privadas. Sin embargo, entre 1955 y 1965 si bien la matrícula de educación superior crece un 38,7\%, la participación relativa del sector privado aumenta del 1 al 8\% (Durham \& Sampaio, 1998, p. 8). De esta manera, se consolida el modelo de acceso abierto a la universidad (sin restricciones ni exámenes de admisión) como un valor asociado a la 


\section{pro.posições}

democratización, concepción que se fortalece por la oposición estudiantil a las políticas restrictivas impulsadas por las dictaduras militares.

Hasta avanzada la mitad del siglo XX, la educación superior no universitaria (terciaria) se concentra fundamentalmente en la formación docente y la educación técnico-profesional representando una pequeña proporción de la matrícula del sector. Entre 1960 y 1980, las instituciones no universitarias (terciarias) — públicas y privadas- llegan a quintuplicarse aunque no logran superar el 20\% de la matrícula de estudiantes del conjunto del nivel (Dirié, 2000). Las reformas educativas de la década de 1990 marcan una segunda ola de expansión de universidades privadas que acompañará también el crecimiento institucional de universidades públicas y de la educación superior no universitaria.

En la actualidad la oferta de educación superior en la Argentina comprende dos circuitos paralelos y segmentados de formación: el universitario (conformado por las universidades nacionales y provinciales financiadas por el aporte del Estado Nacional, y las universidades privadas) y el no universitario (terciario), modalidad predominantemente de formación docente, técnico-profesional y/o mixta entre ambas especialidades. Al igual que la universitaria, la educación superior no universitaria se desarrolla en el sector privado y en el público, siendo en este último caso dependiente de las jurisdicciones, es decir de las provincias y de la Ciudad Autónoma de Buenos Aires. Históricamente, la matrícula del nivel superior universitario es notoriamente más alta que la del no universitario, dado que el primer circuito alcanza una mayor legitimidad social. En los últimos años, la educación universitaria avanza en la oferta y acreditación de ciclos de formación no universitario por lo que el sistema de educación superior se complejiza sin lograr aún consolidar cierta integración y articulación entre ambas modalidades.

Con respecto a la formación universitaria, a partir del retorno de la democracia en la Argentina y la eliminación de las restricciones al ingreso en 1983, las universidades recuperan su tendencia histórica a la expansión matricular, que en los primeros años adopta una verdadera explosión del ingreso, al incrementarse la matrícula en un 30\% (Suasnábar \& Rovelli, 2011). En las siguientes dos décadas, el aumento se sostiene a tasas más o menos similares para entrar en los últimos años en una meseta. Este proceso de ampliación del acceso se refleja en las tasas de escolarización universitaria (neta y bruta) que para el año 2012 


\section{pro.posıções}

alcanzan 19,3\% y 37,8\% respectivamente, mientras que la tasa bruta de escolarización superior se eleva al 53,2\% (Anuario de Estadísticas Universitarias, 2012) ${ }^{4}$.

Cuadro I. Matrícula e instituciones universitarias por sector, 2004-2012

\begin{tabular}{ccccccccc}
\hline & \multicolumn{2}{c}{$\mathbf{1 9 9 6}$} & \multicolumn{2}{c}{$\mathbf{2 0 0 4}$} & \multicolumn{2}{c}{$\mathbf{2 0 0 8}$} & \multicolumn{2}{c}{$\mathbf{2 0 1 2}$} \\
\hline Sector & Matrícula & Instituciones & Matrícula & Instituciones & Matricula & Instituciones & Matrícula & Instituciones \\
Público & 812.308 & 36 & 1.293 .489 & 45 & 1.283 .482 & 48 & 1.442 .286 & 55 \\
Privado & 152.314 & 40 & 233.821 & 55 & 317.040 & 57 & 382.618 & 62 \\
Total & 964.622 & 76 & 1.527 .310 & 100 & 1.600 .522 & 105 & 1.824 .904 & 117 \\
\% & $\mathbf{1 0 0}$ & $\mathbf{1 0 0}$ & $\mathbf{5 8 , 3}$ & $\mathbf{3 1 , 5}$ & $\mathbf{6 5 , 9}$ & $\mathbf{3 8 , 1}$ & $\mathbf{8 9 , 1}$ & $\mathbf{5 3 , 9}$ \\
\hline
\end{tabular}

Fuente: elaboración propia en base a Anuarios de Estadísticas Universitarias 1996, 2004, 2008 y 2012 , Secretaría de Políticas Universitarias, Ministerio de Educación.

En esa dirección, el cuadro I muestra que, entre 1996 y 2012, la matrícula universitaria aumenta un $89,1 \%$, siendo altamente predominante la preferencia por el sector público. Sin embargo, ese acelerado crecimiento no tiene el mismo dinamismo en el sector público que en el privado, ya que este último lo hará a tasas más elevadas que el primero al duplicar ampliamente el número de alumnos durante el período analizado. Por otra parte, si a mediados de los años noventa, el sector público reunía a un $84,2 \%$ del total de la matrícula universitaria, en 2012 su representación disminuye al 79,0\% en favor del crecimiento del sector privado. Al analizar los datos disponibles para el período 2004-2012 se observa la misma tendencia, ya que la matrícula universitaria total crece un 19,4\%, mientras que el sector público aumenta un $11,5 \%$, el privado lo hace en un $63,6 \%$.

Cabe destacar que en América Latina, la privatización de la educación superior constituye una tendencia generalizada, que a partir de la década de 1990 incide diferencialmente en los países de acuerdo a su configuración histórica. En aquellos donde el sector privado posee una larga presencia, la oferta privada se profundiza; mientras que en países como la Argentina -donde hasta mediados del siglo XX esa oferta era inexistente- la expansión del sector, impulsado por las reformas neoliberales, no logra revertir el carácter todavía predominante de las universidades públicas.

\footnotetext{
${ }^{4}$ Los datos son estimados en base a la población de 18 a 24 años del sistema de educación superior de la Argentina.
} 


\section{pro.posições}

ISSN 1980-6248

En el plano del crecimiento institucional, a diferencia de lo ocurrido durante la década de 1990 cuando el sector privado lidera el motor del proceso de diferenciación institucional, en los primeros doce años del nuevo siglo es el sector público el que adopta un papel más importante. Siguiendo el cuadro I, si entre 1996 y 2004 aumentan un 25\% las instituciones del sector público y un 37,7\% las del privado, la tendencia se invierte entre 2004 y 2012 al incrementarse las primeras un 22,2\% y las segundas sólo un 12,7\%.

En los últimos años sobresale la creación de nuevas universidades fundamentalmente públicas. A fines de 2014 se promueve la fundación o bien transformación de anteriores institutos universitarios en once universidades nacionales. De ellas, seis leyes de creación universitaria ya han sido sancionadas y promulgadas. A lo anterior se suman una inusitada ampliación de la oferta académica de las casas de estudio públicas y privadas mediante la proliferación de centros regionales, sedes, subsedes, extensiones áulicas y diversos centros de educación a distancia a nivel nacional. Esta última modalidad se caracteriza por la informalidad de su crecimiento, en virtud de la escasa regulación estatal. De acuerdo a una investigación reciente, entre 1995 y 2012 el número de "extensiones áulicas" se eleva a más de doscientas cincuenta y ocho (Zelaya, 2012).

Cuadro II. Matrícula e instituciones de educación superior no universitaria (terciario), 1996-2012

\begin{tabular}{|c|c|c|c|c|c|c|c|c|}
\hline & \multicolumn{2}{|c|}{1996} & \multicolumn{2}{|c|}{2004} & \multicolumn{2}{|r|}{2008} & \multicolumn{2}{|r|}{2012} \\
\hline Sector & Matrícula & Instituciones & Matrícula & $\begin{array}{c}\text { Institucio } \\
\text { nes }\end{array}$ & Matrícula & Instituciones & Matrícula & Instituciones \\
\hline Público & 233.236 & 797 & 283.433 & 777 & 329.042 & 913 & 473.488 & 992 \\
\hline Privado & 122.851 & 1005 & 228.569 & 1.060 & 278.191 & 1.139 & 294.488 & 1.172 \\
\hline Total & 356.087 & 802 & 512.002 & 1.837 & 607.233 & 2.052 & 767.698 & 2.164 \\
\hline$\%$ & 100 & 100 & 47,7 & 129 & 70,5 & 155,8 & 115,5 & 169,8 \\
\hline
\end{tabular}

Fuente: elaboración propia en base a Anuarios Estadísticos 1996, 2004, 2008 y 2012 de la Dirección Nacional de Información y Evaluación de la Calidad Educativa (DNIECE), Ministerio de Educación.

En relación con la formación superior no universitaria (terciario), corresponde señalar que entre la década de 1960 y mediados de la de 1990 dicho circuito alcanza un vertiginoso crecimiento en la Argentina y en particular en el sector privado. Del cuadro II, se desprende que entre 1996 y 2012 continúa la tendencia expansiva del circuito superior no universitario (terciario), superador del crecimiento universitario, a partir un incremento del 115,5\% de la matrícula y 169,8\% de las instituciones. En ese mismo período, aunque sigue siendo predominante la preferencia por el sector público, la matrícula privada se incrementa un 


\section{pro.posições}

$139,7 \%$ y la pública un $103 \%$. No obstante, las instituciones privadas crecen un 16,6\%, mientras que las públicas aumentan un $24,4 \%$.

Al considerar el período 2004-2012 se observa un crecimiento vigoroso del 49,9\% de la matrícula superior no universitaria (terciario), más acentuado que el 19,4\% de la del circuito universitario durante los mismos años. En contraposición a las anteriores tendencias de crecimiento de la matrícula privada, durante este período es más pronunciado el aumento de la matrícula de instituciones superiores no universitarias (terciario) públicas, en un 67\%, en detrimento del 28,8\% del sector privado. Por otra parte, se produce una ampliación moderada, en un $17,8 \%$, de las instituciones de educación superior no universitaria (terciario) y continúa la tendencia del período 2004-2012 orientada hacia un mayor crecimiento institucional del sector público en un $27,6 \%$ y en menor medida, en un 10,5\% del privado.

\section{Ampliación, oportunidades de acceso y desigualdad en la Educación Superior}

La evolución de los estudiantes entre 18 y 30 años que asisten a la educación superior (universitaria y no universitaria) entre 2004 y 2012 según quintil de ingreso per cápita de los hogares argentinos es presentada en el Cuadro III, el cual permite cuantificar y poner en perspectiva en qué medida el sistema se ha ido abriendo a los grupos de menores ingresos de la sociedad ${ }^{5}$.

5Para el relevamiento estadístico decidimos tomar un rango de población más amplio en edad - de 18 a 30 añosque los utilizados por la OCDE -de 20 a 24 años- y el Ministerio de Educación de la Argentina -18 a 24 añospara medir la tasa de escolarización del sistema de educación superior. El criterio escogido privilegia una medición más amplia de la población que circula por el sistema de educación superior, atendiendo al amplio espectro etario que resulta una característica idiosincrática del caso argentino. 


\section{pro.posıções}

ISSN 1980-6248

Cuadro III. EPH - Estudiantes entre 18 y 30 años que asisten a un establecimiento de educación superior por quintil de ingreso per cápita familiar

\begin{tabular}{cccccccccc}
\hline Quintiles & $\mathbf{2 0 0 4}$ & $\mathbf{2 0 0 5}$ & $\mathbf{2 0 0 6}$ & $\mathbf{2 0 0 7}$ & $\mathbf{2 0 0 8}$ & $\mathbf{2 0 0 9}$ & $\mathbf{2 0 1 0}$ & $\mathbf{2 0 1 1}$ & $\mathbf{2 0 1 2}$ \\
\hline $\mathbf{1}$ & $10,76 \%$ & $11,01 \%$ & $10,06 \%$ & $12,32 \%$ & $13,11 \%$ & $14,61 \%$ & $14,57 \%$ & $15,08 \%$ & $15,62 \%$ \\
$\mathbf{2}$ & $18,37 \%$ & $18,37 \%$ & $18,58 \%$ & $19,97 \%$ & $17,12 \%$ & $18,10 \%$ & $17,72 \%$ & $20,10 \%$ & $20,56 \%$ \\
$\mathbf{3}$ & $20,58 \%$ & $21,98 \%$ & $21,90 \%$ & $20,20 \%$ & $22,91 \%$ & $22,15 \%$ & $22,78 \%$ & $21,29 \%$ & $22,30 \%$ \\
$\mathbf{4}$ & $25,75 \%$ & $25,42 \%$ & $25,66 \%$ & $24,49 \%$ & $25,69 \%$ & $24,31 \%$ & $24,46 \%$ & $24,56 \%$ & $24,64 \%$ \\
$\mathbf{5}$ & $24,54 \%$ & $23,22 \%$ & $23,80 \%$ & $23,01 \%$ & $21,17 \%$ & $20,83 \%$ & $20,48 \%$ & $18,97 \%$ & $16,88 \%$ \\
\hline
\end{tabular}

Fuente: elaboración propia sobre datos del Indec, EPH (2004-2012).

En 2004, un 10,76\% de los casos analizados de jóvenes pertenecientes al primer quintil se encuentra cursando alguna carrera de educación superior mientras que en 2008, la representación de estos sectores se incrementa, alcanzando un 13,11\% de asistencia en 2008 y un $15,62 \%$ en 2012 , lo que sin duda representa un avance en términos de oportunidades de acceso para los grupos de menores ingresos del país ${ }^{6}$.

\begin{tabular}{|c|c|c|c|c|c|c|}
\hline \multicolumn{7}{|c|}{$\begin{array}{c}\text { Cuadro IV. EPH - Estudiantes entre } 18 \text { y } 30 \text { años que asisten a un establecimiento } \\
\text { de educación superior según tipo de educación y quintil de ingreso per cápita } \\
\text { familiar }\end{array}$} \\
\hline & \multicolumn{2}{|c|}{2004} & \multicolumn{2}{|c|}{2008} & \multicolumn{2}{|c|}{2012} \\
\hline Quintiles & Terciario & Universitario & Terciario & Universitario & Terciario & Universitario \\
\hline 1 & $27,28 \%$ & $72,72 \%$ & $27,35 \%$ & $72,65 \%$ & $28,32 \%$ & $71,68 \%$ \\
\hline 2 & $22,57 \%$ & $77,43 \%$ & $26,26 \%$ & $73,74 \%$ & $24,15 \%$ & $75,85 \%$ \\
\hline 3 & $20,20 \%$ & $79,80 \%$ & $23,71 \%$ & $76,29 \%$ & $22,71 \%$ & $77,29 \%$ \\
\hline 4 & & $83,95 \%$ & $20,49 \%$ & $79,51 \%$ & $17,75 \%$ & $82,25 \%$ \\
\hline 5 & $13,05 \%$ & $86,95 \%$ & $13,85 \%$ & $86,15 \%$ & $19,89 \%$ & $80,11 \%$ \\
\hline
\end{tabular}

Fuente: elaboración propia sobre datos de Indec, EPH (2004-2012).

Ahora bien, el cuadro IV evidencia que el acceso a educación superior en Argentina se encuentra diferenciado según el nivel de ingresos de las familias de los estudiantes y el tipo de educación superior, universitaria o no universitaria (terciaria) a la que acceden. Al respecto, en 2004 la proporción de estudiantes que asisten a un establecimiento de educación superior no

${ }^{6}$ Cabe destacar que el estudio realizó un relevamiento paralelo de estudiantes entre 18 y 25 años que asisten a un establecimiento de Educación superior, el cual no arroja grandes diferencias en el crecimiento de la participación de los distintos quintiles respecto al recorte de la población de 18 a 30 que aquí analizamos. En ese sentido, en 2004 un 11,26\% de los casos analizados de jóvenes pertenecientes al primer quintil se encuentra cursando alguna carrera de educación, alcanzando un 13,86\% de asistencia en 2008 y un 17,22\% en 2012. Al considerar a los sectores de mayores ingresos, puede observarse el pasaje desde una representación de los jóvenes que asisten a la educación superior del 22,31\% en 2004 al 19,42 \% en 2008 y finalmente cae al 14,22\% en 2012. 


\section{pro.posições}

ISSN 1980-6248

universitaria (terciaria) del primer quintil es del 27,28\%; mientras que del quinto quintil la proporción que acude es del 13,05\%. Por el contrario, ese mismo año la proporción de estudiantes que asisten a un establecimiento de educación superior universitaria del primer quintil es del 72,72\%; mientras del quinto quintil concurre el 86,95\%. La tendencia para los estudiantes de sectores populares se mantiene sin grandes modificaciones en 2008; mientras que en 2012 crece alrededor de un punto la proporción de asistencia a un establecimiento de educación superior no universitaria (terciaria) en detrimento de la proporción de su participación en la educación superior universitaria ${ }^{7}$.

Cabe destacar que un estudio reciente afirma que en algunos territorios donde existe una variada oferta de universidades públicas, como por ejemplo la Ciudad Autónoma de Buenos Aires y La Plata, suele no haber demasiadas diferencias en los datos de acceso de los distintos quintiles a la educación universitaria y no universitaria (terciario) o bien tener menos alumnos en esta última oferta (Raverta, 2012, p. 33).

\begin{tabular}{|c|c|c|c|c|c|c|}
\hline $\begin{array}{r}\text { Cuadro V } \\
\text { de Edu }\end{array}$ & $\begin{array}{l}\mathrm{PH}-\mathrm{I} \\
\text { ión Su }\end{array}$ & $\begin{array}{l}\text { antes en } \\
\text { (univer }\end{array}$ & $\begin{array}{l}3 \text { y } 30 \text { añ } \\
\text { o y no u. }\end{array}$ & $\begin{array}{l}\text { e asister } \\
\text { sitario) } \mathrm{s} \\
\text { ta famili }\end{array}$ & $\begin{array}{l}\text { n es } \\
\text { n sector } \mathrm{c}\end{array}$ & $\begin{array}{l}\text { cimiento } \\
\text { stión y }\end{array}$ \\
\hline & & & 20 & & & \\
\hline Quintiles & Público & Privado & Público & Privado & Público & Privado \\
\hline 1 & $86,23 \%$ & $13,48 \%$ & $82,12 \%$ & $17,84 \%$ & $82,38 \%$ & $17,60 \%$ \\
\hline 2 & $86,41 \%$ & $13,50 \%$ & $79,59 \%$ & $20,40 \%$ & $81,64 \%$ & $18,21 \%$ \\
\hline 3 & $82,69 \%$ & $17,26 \%$ & $76,89 \%$ & $22,98 \%$ & $76,69 \%$ & $23,18 \%$ \\
\hline 4 & $79,11 \%$ & $20,89 \%$ & $72,62 \%$ & $27,35 \%$ & $70,81 \%$ & $29,18 \%$ \\
\hline 5 & $70,01 \%$ & $29,82 \%$ & $66,3 \%$ & $33,59 \%$ & $63,55 \%$ & $36,37 \%$ \\
\hline
\end{tabular}

Fuente: elaboración propia sobre datos de Indec, EPH (2004-2012).

Paralelamente, el cuadro $\mathrm{V}$ presenta la distribución de los estudiantes según el tipo de institución de educación superior, diferenciando entre las universidades pertenecientes al sector público y al privado. Cabe destacar que en cada uno de los quintiles de pertenencia la representación de los estudiantes es mayoritaria -más de un 63\%- en las instituciones públicas, ya sean de nivel superior universitario o no universitario (terciario). Sin embargo, si

\footnotetext{
7 Resulta llamativo el crecimiento de la participación de estudiantes del quinto quintil en la educación superior terciaria, la cual aumenta desde el 13,05\% en 2004 al 19,89\% en 2012. Si bien escapa a las posibilidades de esta investigación poder realizar estudios más específicos sobre este cambio, consideramos que la ampliación de ofertas educativas más exclusivas, relacionadas con nichos de oportunidades en el mercado laboral y redes de relaciones de ámbitos laborales específicos, inciden en la opción de los estudiantes de este quintil por carreras de formación de grado que tradicionalmente no se encuentran en el ámbito universitario.
} 


\section{pro.posições}

en 2004 el porcentaje de estudiantes del primer quintil pertenecientes a establecimientos de educación superior privados es del 13,48\%; en 2008 aumenta hasta el 17,84\% y se mantiene en un $17,60 \%$ en 2012. Por su parte, en el quinto quintil dicha cifra alcanza un $29,82 \%$ en 2004 , mientras que en 2012 aumenta al 36,37\% (cuadro V). Al respecto, otros estudios afirman que el crecimiento de la matrícula del sector privado en el ámbito universitario (sin tomar en cuenta a las instituciones terciarias no universitarias) resulta más intenso hasta el 2008. A partir de ese año, esa preferencia ingresa en una meseta y se observa un mayor crecimiento del sector estatal en detrimento del privado: el primero crece un 5,5\% y un 4,1\% el segundo (Botinelli, 2013).

Siguiendo el análisis, este cambio favorable al subsistema universitario público estaría vinculado, en parte, con una nueva oferta de instituciones públicas y carreras de grado en territorios que se encontraban alejados de los ámbitos académicos. En particular, varias de las universidades nacionales creadas en los últimos años se orientan a captar estudiantes que son en su familia la primera generación que accede a la universidad. En particular, el estudio destaca que el crecimiento de estudiantes en el área del Conurbano de la Provincia de Buenos Aires, una de las zonas con nichos poblacionales más vulnerables económicamente del país, pertenece a instituciones universitarias públicas.

De manera panorámica y ante el crecimiento del sistema de educación superior, las tendencias anteriores pueden explicarse tanto como parte de los procesos de diferenciación de la demanda educativa como también resultado -al menos hasta 2008- de cierto crecimiento económico (Kessler, 2014). Ciertamente, a lo largo del período estudiado se observa un crecimiento en la participación de los estudiantes tanto de mayores como de menores ingresos (aunque de manera diferencial) en la educación superior privada aunque, como se dijo anteriormente, a partir de 2008 la representación de los estudiantes que asisten a la educación superior se mantiene relativamente estable en los primeros quintiles de ingreso. De todas formas, desde un plano institucional y político-educativo cabría preguntarse cuáles son los factores que inciden en que un sector de jóvenes provenientes de los sectores de menores ingresos opten por pagar sus estudios superiores en instituciones privadas ante la existencia de una oferta amplia, gratuita y legitimada socialmente de educación universitaria. Y a la vez, indagar en los dispositivos y estrategias institucionales que en los últimos años se generan desde distintas instituciones públicas y programas gubernamentales para recuperar dicha matrícula. 


\section{pro.posições}

ISSN 1980-6248

En paralelo a la cuestión de la igualdad en el acceso a la educación superior, comienza a problematizarse a nivel regional y en particular en la Argentina, la igualdad de resultados. Al analizar la tasa de graduación en educación superior para América Latina en 2007, Argentina alcanza un 14\%, cercano a Venezuela y Chile, países que poseen índices de alrededor del 15\% y por arriba de Colombia con un 11\%. Por su parte, Brasil y México, obtienen el 19\% respectivamente, lejos de Cuba, la cual llega al 21\% (Unesco, 2007).

En líneas generales cabe destacar que entre 2004 y 2012 los graduados de títulos de pre-grado y grado en el ámbito universitario argentino (público y privado) aumentan un 31,5\% (Anuario de Estadísticas Universitarias, 2012). De todas formas, con respecto al 2004, la tasa de graduados en el ámbito universitario privado en 2012 se amplía un 80,8\%, mientras que la del universitario público sólo se incrementa un 15,7\% (Anuario de Estadísticas Universitarias, 2012).

Cuadro VI. EPH - Graduados de Educación Superior según quintil de ingreso per cápita familiar

\begin{tabular}{llllllllll}
\hline TOTAL & $\mathbf{2 0 0 4}$ & $\mathbf{2 0 0 5}$ & $\mathbf{2 0 0 6}$ & $\mathbf{2 0 0 7}$ & $\mathbf{2 0 0 8}$ & $\mathbf{2 0 0 9}$ & $\mathbf{2 0 1 0}$ & $\mathbf{2 0 1 1}$ & $\mathbf{2 0 1 2}$ \\
\hline Quintil 1 & $5,02 \%$ & $4,54 \%$ & $4,06 \%$ & $4,73 \%$ & $5,87 \%$ & $5,83 \%$ & $5,28 \%$ & $5,44 \%$ & $6,31 \%$ \\
Quintil 2 & $9,80 \%$ & $11,26 \%$ & $9,75 \%$ & $10,60 \%$ & $9,33 \%$ & $9,43 \%$ & $10,17 \%$ & $12,47 \%$ & $12,15 \%$ \\
Quintil 3 & $15,18 \%$ & $16,34 \%$ & $15,73 \%$ & $15,22 \%$ & $16,54 \%$ & $15,59 \%$ & $18,39 \%$ & $17,50 \%$ & $18,02 \%$ \\
Quintil 4 & $25,81 \%$ & $27,43 \%$ & $26,18 \%$ & $24,83 \%$ & $25,62 \%$ & $25,78 \%$ & $25,31 \%$ & $25,11 \%$ & $25,78 \%$ \\
Quintil 5 & $44,18 \%$ & $40,43 \%$ & $44,29 \%$ & $44,62 \%$ & $42,63 \%$ & $43,37 \%$ & $40,84 \%$ & $39,47 \%$ & $37,74 \%$ \\
\hline
\end{tabular}

Fuente: elaboración propia sobre datos del Indec, EPH (2004-2012)

Paralelamente, al considerar la igualdad de resultados en educación superior en la Argentina de acuerdo a la representación de los graduados según quintil de ingreso per cápita familiar, se observa que durante el período 2004-2012 aumenta del 5 al 6,3\% para los jóvenes de los primeros quintiles. A su vez, la representación de egresados del segundo quintil crece del 9,8 al $12,2 \%$, lo cual corrobora la tendencia anterior. 


\section{pro.posıções}

ISSN 1980-6248

Cuadro VII. EPH - Graduados de educación superior según tipo de institución y quintil de ingreso per cápita familiar

\begin{tabular}{ccccccc}
\hline & \multicolumn{2}{c}{$\mathbf{2 0 0 4}$} & \multicolumn{2}{c}{$\mathbf{2 0 0 8}$} & \multicolumn{2}{c}{$\mathbf{2 0 1 2}$} \\
\hline Quintiles & Terciario & Universitario & Terciario & Universitario & Terciario & Universitario \\
$\mathbf{1}$ & $70,42 \%$ & $29,58 \%$ & $71,76 \%$ & $27,86 \%$ & $66,52 \%$ & $33,28 \%$ \\
$\mathbf{2}$ & $68,28 \%$ & $31,72 \%$ & $68,03 \%$ & $30,89 \%$ & $63,60 \%$ & $35,63 \%$ \\
$\mathbf{3}$ & $61,14 \%$ & $38,86 \%$ & $57,29 \%$ & $42,22 \%$ & $58,30 \%$ & $41,41 \%$ \\
$\mathbf{4}$ & $53,99 \%$ & $45,84 \%$ & $49,32 \%$ & $49,50 \%$ & $44,97 \%$ & $54,13 \%$ \\
$\mathbf{5}$ & $32,55 \%$ & $66,32 \%$ & $32,37 \%$ & $66,64 \%$ & $34,41 \%$ & $62,33 \%$ \\
\hline
\end{tabular}

Fuente: elaboración propia sobre datos del Indec, EPH (2004-2012)

Siguiendo el análisis, la tasa de graduados de educación superior según tipo de institución y quintil de ingreso per cápita familiar también aporta otro dato interesante que se corresponde con la anterior descripción sobre el acceso. Del cuadro VII se observa la tendencia a una mayor representación en el egreso de los quintiles de menores ingresos en la educación superior no universitaria (terciaria) en comparación con los quintiles más altos: en 2012 alcanza un 66,5\% para los primeros y 34,4\% para los segundos. En el mismo año, esta relación es inversa en el ámbito universitario: en un 33,2\% están representados los quintiles de menores ingresos y en un $62,3 \%$ los de mayores ingresos.

\section{Interjuegos entre la matrícula de la escuela media y el acceso a superior}

Llegados a este punto del trabajo, interesa explorar en qué medida y de qué manera las lógicas de expansión del nivel medio inciden en el crecimiento del acceso de los sectores populares en la educación superior en la Argentina.

Sobre la expansión de la matrícula en la escuela secundaria, corresponde señalar que a partir de la década de 1990, el sistema educativo lleva a cabo una incorporación masiva de estudiantes, principalmente en el nivel secundario. La tasa neta de escolarización secundaria pasa del 63,3\% en 1980 al 71,8\% en 1991, se incrementa al 81,3\% en 2001 y alcanza en 2011 un $84,4 \%$. 


\section{pro.posıções}

Cuadro VIII. Tasas de escolarización nivel medio 1980, 1991, 2001 y 2011

\begin{tabular}{ccccc}
\hline & $\mathbf{1 9 8 0} *$ & $\mathbf{1 9 9 1 *}$ & $\mathbf{2 0 0 1} * *$ & $\mathbf{2 0 1 1} * *$ \\
\hline $\begin{array}{c}\text { Población } \\
\mathbf{1 3} \text { a 17 años }\end{array}$ & $63,3 \%$ & $71,8 \%$ & $81,3 \%$ & $84,4 \%$ \\
\hline
\end{tabular}

* Fuente: Diniece (2003). Tendencias recientes de la escolarización y terminalidad del nivel medio de enseñanza.

** Fuente: Siteal, IIPE - Unesco / OEI (2001-2011) en base a: Argentina - EPH del Indec,.

Cabe destacar que en el año 2006, la sanción de la Ley de Educación Nacional $\left(\mathrm{N}^{\circ}\right.$ 26.206) establece como finalidad garantizar la alfabetización y el cumplimiento de la obligatoriedad escolar hasta la finalización del nivel secundario. En ese marco, el Ministerio de Educación lanza recientemente distintos programas, a fin de posibilitar ese derecho educativo $^{8}$. Sin embargo, la disminución en el ritmo de crecimiento de la tasa de escolarización del nivel medio en los últimos años podría explicarse tanto por la cercanía de la meta de universalización del nivel, como por la dificultad de incorporar en la escuela a un núcleo poblacional en extrema exclusión.

Cuadro IX. EPH - Estudiantes entre 13 y 17 años que asisten a un establecimiento de nivel medio según sector de gestión y quintil de ingreso per cápita familiar

\begin{tabular}{ccccccc}
\hline \multicolumn{2}{c}{ 2006 } & \multicolumn{2}{c}{$\mathbf{2 0 0 9}$} & \multicolumn{2}{c}{$\mathbf{2 0 1 2}$} \\
\hline $\begin{array}{c}\text { Sector } \\
\text { Quintiles }\end{array}$ & Público & Privado & Público & Privado & Público & Privado \\
$\mathbf{1}$ & & & & & & \\
$\mathbf{2}$ & $87,00 \%$ & $12,96 \%$ & $88,67 \%$ & $11,26 \%$ & $87,36 \%$ & $12,56 \%$ \\
$\mathbf{3}$ & $74,72 \%$ & $25,23 \%$ & $73,99 \%$ & $25,89 \%$ & $72,67 \%$ & $27,19 \%$ \\
$\mathbf{4}$ & $64,88 \%$ & $35,01 \%$ & $61,72 \%$ & $38,28 \%$ & $58,17 \%$ & $41,14 \%$ \\
$\mathbf{5}$ & $50,02 \%$ & $49,53 \%$ & $48,45 \%$ & $51,55 \%$ & $45,51 \%$ & $54,49 \%$ \\
\hline
\end{tabular}

Fuente: elaboración propia sobre datos del Indec, EPH (2006-2012)

${ }^{8}$ Si bien excede las posibilidades de análisis de este trabajo, el Plan de Finalización de Estudios Primarios y Secundarios para Jóvenes y Adultos (Plan FinES) se destaca como uno de los programas más extendidos a lo largo del país que procura desde su implementación en 2008 ampliar el acceso, la permanencia y egreso a la educación secundaria. 


\section{pro.posições}

Por otra parte, al analizar el porcentaje de estudiantes de entre 13 y 17 años que asisten a un establecimiento de nivel medio según sector de gestión y quintil de ingreso se observa que en 2012 la representación de los estudiantes es mayoritaria en las instituciones públicas más de un $87 \%$ y un $72 \%$ - sólo en los primeros y segundos quintiles, respectivamente. En los estratos medios, representados en el tercer quintil, resulta un poco más pareja la participación de estudiantes en las escuelas públicas, incluso con mayor preferencia y en las privadas: 58,1\% y $41,1 \%$, respectivamente. Sin embargo, durante los seis años transcurridos entre 2006 y 2012, la representación de estudiantes que más crece en la escuela privada, asciende del 35 al 41\%, es la del tercer quintil. Finalmente, la tendencia comienza a profundizarse acentuadamente al observar la representación del cuarto y quinto quintil en la educación privada: el primero a través de una participación del 54,49\% y el segundo, mediante el 62,82\%.

Así, el moderado crecimiento en la tasa de escolarización del nivel secundario (cuadro VIII) y el limitado aumento de la representación de los quintiles de menores ingresos en la educación superior inciden en las posibilidades y condiciones de acceso de los sectores populares a la educación superior. En efecto, la dificultad de avanzar en la universalización del nivel secundario desacelera la demanda de acceso a la educación superior, mientras que el leve incremento de la representación de los quintiles de menores ingresos en este último nivel se corresponde en buena medida con su ingreso a un segmento de universidades e instituciones terciarias privadas de mercado.

\section{Consideraciones finales}

El cruce entre las dinámicas de expansión de la educación superior y secundaria en la Argentina, particularmente entre 2004 y 2012 y la composición social de la matrícula, su configuración por circuitos de formación y sectores permite bosquejar algunas reflexiones finales en torno a la problemática de la desigualdad.

En el plano institucional y en contraste con lo ocurrido durante la década de 1990, las instituciones del sector público del conjunto de la educación superior (universitaria y no universitaria) adquieren un mayor crecimiento entre 2004 y 2012 en comparación con las del sector privado. No obstante, proliferan en ambos sectores instituciones con misiones, orientaciones, propósitos y prestigio diversos. 


\section{pro.posições}

Por otra parte, se observa un crecimiento global de la matrícula de la educación superior, la que en 2012 sigue la tendencia histórica de mayor concentración de estudiantes en el sector universitario $(70,4 \%)$ en detrimento del no universitario (terciario). Sin embargo, entre 2004 y 2014 la matrícula de este último circuito, con menor legitimidad social, adopta un ritmo más vigoroso de crecimiento en comparación con el universitario. Sumado a lo anterior, si bien sigue siendo predominante la preferencia por las instituciones de educación superior públicas, tanto universitarias como no universitarias, la matrícula universitaria privada crece con gran dinamismo, lo cual profundiza la diferenciación institucional.

Con respecto a la ampliación de la composición social de la matrícula de la educación superior hacia los grupos sociales de menores ingresos, cabe destacar que aumenta entre 2004 y 2012 del 10,76 al 15, 62\%. Al respecto, consideramos que el carácter moderado de la expansión se vincula principalmente con cierto amesetamiento de la tasa de escolarización secundaria. Asimismo, dichos sectores adquieren una presencia preponderante en el sector público y si bien sobresale su participación en el circuito universitario, crece comparativamente respecto de los otros quintiles su representación en el sector no universitario (terciario). De allí que la tendencia hacia la profundización de circuitos diferenciados según ingresos en el nivel secundario incida, en cierta medida, en la selectividad de las lógicas de expansión de la educación superior, al ampliar los sectores sociales de menores ingresos su participación en circuitos de formación con menor legitimación social.

Finalmente, si bien en un escenario de acceso "amplio" a la educación superior, la tasa de graduados resulta moderada en comparación con otros países de la región, entre 2004 y 2012 aumenta del 5,02\% al 6,31\% la representación de los primeros quintiles, particularmente en el nivel no universitario.

Con todo, el modelo de educación superior argentino continúa siendo predominantemente público, dado que en ese sector se concentra más del $63 \%$ de representación de todos los quintiles de ingreso (cuadro V). No obstante, los distintos sectores sociales aumentan su participación en la educación superior privada hasta el año 2008, cuando comienza a estancarse la representación de los quintiles de menores ingresos. La tendencia es, sin embargo, constante y creciente entre los sectores de mayores ingresos, fenómeno que se intensifica en el nivel secundario. 


\section{pro.posıções}

En relación con la desigualdad, principalmente la universidad y en menor medida las instituciones de educación superior no universitarias (terciarios) siguen siendo espacios educativos favorables a los más favorecidos socioeconómicamente. En ese sentido, en los últimos años tanto desde el gobierno central como desde las instituciones de educación superior se llevan adelante distintas iniciativas que procuran generar mejores condiciones para garantizar la igualdad de acceso, a través de becas y ayudas económicas, como también la igualdad de oportunidades mediante la "compensación" o la "neutralización" de los condicionamientos de origen sociocultural de los estudiantes. Estas últimas políticas, nucleadas en torno a la idea de ampliación del derecho a la educación superior o acción afirmativa acarrean diversas críticas acerca de su contribución al aumento de la selectividad, la competencia y las estrategias individuales de los sectores menos favorecidos al interior del sistema de educación superior.

Queda pendiente el debate sobre los distintos instrumentos de política educativa que en nuestro país apunten a reducir las brechas entre las condiciones y posibilidades educativas de los más débiles y los más pobres socialmente. Asimismo, resulta central la necesidad de elaborar y sostener en el tiempo herramientas estadísticas que permitan ir evaluando tales brechas educativas, a fin de contribuir con la toma de decisión pública. 


\section{pro.posıções}

ISSN 1980-6248

\section{Bibliografía}

Aponte-Hernández, E. (2008). Desigualdad, inclusión y equidad en la educación superior en América Latina y el Caribe: tendencias y escenarios alternativos en el horizonte 2021. In IESALC/UNESCO. Tendencias la educación superior en América Latina y el Caribe. Santiago de Chile.

Barefoot, B. (2000, jan./feb.). The first-year experience. Are we making it any better? About Campus, volumen 4, número 6, pp. 12-18.

Barefoot, B. O. (2005). Current institutional practices in the first college year. In M. L. Upcraft, J. N. Gardner, \& B. O. Barefoot (Comp.), Challenging and supporting first year student. A handbook for proving first year in college. San Francisco: Jossey-Bass.

Botinelli, L. (2013). El debate sobre el crecimiento reciente de la educación privada. Buenos Aires: Diniece, Ministerio de Educación.

Boudon, R. (1974). La desigualdad de oportunidades: La movilidad social en las sociedades industriales. España: Laia.

Bourdieu, P. (1970). La reproducción. Elementos para una teoría del sistema de enseñanza. Barcelona, Laia.

Bourdieu, P., \& Passeron, J.-C. (2003). Los herederos. Los estudiantes y la cultura (p. 190, 1a ed.). Buenos Aires: Siglo XXI.

Chiroleu, A. (2009a). La inclusión de la educación superior como política pública: tres experiencias en América Latina. Revista Iberoamericana de Educación, 48, pp. 1-15.

Chiroleu, A. (2009b). Políticas públicas de inclusión en la educación superior: Los casos de Argentina y Brasil. Pro-Posições, 20(2), pp. 141-166.

Chiroleu, A. (2012). Expansión de oportunidades, inclusión y democratización universitaria. In A. Chiroleu, C. Suasnábar, \& L. Rovelli. Política universitaria en la Argentina: revisando viejos legados en busca de nuevos horizontes. Los Polvorines: Universidad Nacional de General Sarmiento, IEC-CONADU.

Collins, R. (1989). La sociedad credencialista. Sociología histórica de la educación y la estratificación. Madrid: Akal. 


\section{pro.posıções}

Duarte, B. (2005). De aspirantes a alumnos. Una clasificación de los sistemas universitarios de admisión y su aplicación a las universidades nacionales de la Argentina. In S. Gvirtz, \& A. Camou (Comp.), La universidad Argentina en discusión: sistemas de ingreso, financiamiento y evaluación de la calidad. Buenos Aires: Gránica

Dubet, F. (2010). Repensar la Justicia Social. Contra el mito de la igualdad de oportunidades. Buenos Aires: Siglo XXI.

Dubet, F. (2012, mayo/junio). Los límites de la igualdad de oportunidades. Nueva Sociedad, 239, pp. $42-50$.

Durham, E. R., \& Sampaio, H. (1998). O setor privado na América Latina: uma análise comparativa (Documento de trabalho, 3, pp. 7-37). São Paulo: Núcleo de Pesquisas sobre Ensino Superior Universidade de São Paulo, Nupes.

Kessler, G. (2014). Controversias sobre la desigualdad. Argentina 2003-2013. Buenos Aires: Fondo de Cultura Económica.

Raverta, C. M. (2012). Estudiantes universitarios en la argentina: un estudio exploratorio en base a la Encuesta Permanente de Hogares. La Plata: Departamento de Sociología, Facultad de Humanidades y Ciencias de la Educación, Universidad Nacional de La Plata.

Siteal. (2005). La educación superior en América Latina: acceso, permanencia y equidad. IIPE/UNESCO/OEI. Extractado en diciembre de 2014, de: http://www.siteal.iipeoei.org/sites/default/files/educacion_superior.pdf

Suasnabar, C., \& Rovelli, L. (2011, diciembre). Políticas universitarias en Argentina: entre los legados modernizadores y la búsqueda de una nueva agenda. Revista de Innovación Educativa, 11(57), pp. 21-30. México, DF: Instituto Político Nacional, Secretaría de Educación Pública.

Tinto, V. (2004). Student retention and graduation. Facing the truth living with the consequences. The Pell Institute for the Study of Opportunity in Higher Education. Extractado en diciembre de 2014, de: http:/ files.eric.ed.gov/fulltext/ED519709.pdf

Trow, M. (2005). Reflections on the transition from elite to mass to universal access: forms and phases of higher education in modern societies since WWII. In P. Altbach (Ed.), International Handbook of Higher Education. Kluwer. 


\section{pro.posıções}

Zelaya, M. (2012, maio/ago.). La expansión de universidades privadas en el caso argentino. Pro-Posições, 23(2), pp. 179-194.

\section{Fuentes consultadas}

Conferencia Regional de Educación Superior (Cres). (2008). Declaración final de la Conferencia Regional de Educación Superior, Cartagena, Colombia.

Dirección Nacional de Información y Evaluación de la Calidad Educativa (Diniece). (1996, 2004, 2008, 2012). Ministerio de Educación. Anuario Estadístico Educativo, Buenos Aires.

Dirección Nacional de Información y Evaluación de la Calidad Educativa (Diniece). (2003). Ministerio de Educación. Tendencias recientes de la escolarización y terminalidad del nivel medio de enseñanza, Buenos Aires.

Dirié, C. (Coord.). (2002). Mapa de la Oferta de Educación Superior en la Argentina del 2000. Secretaría de Educación Superior, Ministerio de Educación. Extractado en diciembre de 2014, de: http://www.oei.es/homologaciones/INFORMEFinal.pdf

Indec. (2004-2012). La Nueva Encuesta Permanente de Hogares de Argentina. Buenos Aires: Indec.

Siteal, IIPE-Unesco/OEI (2001-2011). Tasa Neta de Escolarización Secundaria para Argentina. Disponible en: http://www.siteal.iipe-oei.org/base_de_datos/consulta?i=3\#. Consulta: febrero de 2015

Secretaría de Políticas Universitarias, Ministerio de Educación. (1996, 2004, 2008, 2012). Anuario de Estadísticas Universitarias, Buenos Aires.

Unesco. (2007). Compendio de la Educación Superior. Comparación de las estadísticas de educación superior en el mundo. Montreal: Instituto de Estadística.

Submetido à avaliação em 4 de fevereiro de 2015; aprovado para publicação em 9 de novembro de 2015. 
